\title{
The Research of Order Prediction Model for Textile Machinery Manufacturing Enterprise Based on Customer Demand
}

\author{
Xixing $\mathrm{Li}^{1, \text { a }}$,Shunsheng Guo ${ }^{1, \mathrm{~b}}$, Lei Wang ${ }^{1, \mathrm{c}}$, Zhen $\mathrm{Li}^{2, \mathrm{~d}}$ \\ ${ }^{1}$ School of Mechanical and Electronic Engineering, Wuhan University of Technology, Wuhan \\ 430070, China \\ ${ }^{2}$ School of Management, Henan University of Technology, Zhengzhou 450000, China \\ ali_xi_xing@126.com, bguoshunsheng@263.net, cwanglei9455@126.com, ${ }^{\mathrm{d}}$ lizhenwxh@163.com,
}

Keywords: Order prediction, Improved time series, Customer demand

\begin{abstract}
With the development of information technology, textile machinery manufacturing enterprises (TMME) are facing the new situation of "Two Integration", in order to better responding to market customer demand (CD), the order prediction is becoming more and more important. The background of product model of TMME is different from other manufacturing enterprise, the CD information about TMME is full of diversity, fuzziness and concealment. In this paper, an order prediction model for TMME is constructed by combining the CD with improved time series, also it has been applied and verified in an actual enterprise.
\end{abstract}

\section{Introduction}

At present, the order prediction (OP) has obtained many researches and much attention of scholars in home and abroad, they have taken deeper researches for core manufacturing enterprise based on different forecasting methods. Lundholm [1] used Mono-regression to forecast the sales of retail industry and proposed an action model for growth rate of sales. Wang [2] constructed a forecast method based on moving average and least squares of seasonal factor by comparing the different forecast method of aviation manufacturing industry. Liu [3] combined the time series with multiple regression and got a useful forecast of customer demand overall supply chain. Gong [4] has taken research and application of order prediction model (OPM) based on the traditional minimum two orthogonal multiplication algorithms, also she optimized the model by using improved particle swarm optimization algorithm. Wang [5] proposed three methods of Correct, MA, EWMA for corresponding the time series demand function model ARIMA $(1,0,0)$, ARIMA $(0,0$, 1), ARIMA (1, 0,1), and verified the effect of improved prediction method that under bullwhip effect on the order quantity and actual demand. Gao [6] took the study that based on SVM of product demand prediction, and concluded the great advantage of SVM in product demand prediction by comparing with other prediction methods. Dong [7] has successfully forecasted the order number of product by using improved GP algorithm and established the application model.

In the current market development background of increasing competition, the customer demand is changing more and more quicklyand some prediction researches are always focus on the historical orders data and ignore the customer demand is one key factor in the process of forecasting. This study combines the CD with improved time series to construct a new prediction model, and it is organized as follows: Section 2 mainly analysis the mechanism of OPM; in Section 3, an improved order prediction model (Imp_OPM) is built and a case analysis has been taken; Section 4 draws the conclusion.

\section{The mechanism of OPM}

Order prediction is actually the process of summarizing the different, complex, fuzzy data and getting effective knowledge. According to the characteristics of the customer demand of TMME which are usually implicit, diversity, priority and similarity, the step of order prediction of textile machinery enterprise can be divided as follows: 
(1) Making prediction target and clearing the prediction object. The OP of TMME is taken the sales order as the prediction object, the purpose of making an effective purchasing plan which is based on the actual situation of enterprise, realizing the stock optimization and early warning, quickly responding to customer demand and taking the preemptive opportunities as prediction target.

(2) Collection and analysis of data. At present, with the development and perfection of the supply chain management and the ERP management system used in the TMME, the information sharing platform is changed into more wider and reality, the process of management system and data storage are becoming unified and standard and which provide a solid database for the OPM.

(3) Selection and implementation of order prediction method (OP_M). On the basis of order prediction target and prediction period, and with the combination of TMME's own characteristics and demands, determining the finial prediction method which is mainly about decompose method that based on the improved time series, supplemented by correction of qualitative prediction method.

(4) Analysis and evaluation of prediction result. The evaluation of prediction result is mainly focus on the process of prediction method and tests the accuracy by comparing the prediction result with actual value. The evaluation factors often used are average percentage error, deviation rate and so on.

(5) Summary report of OPM. The report contains prediction process, prediction principle and prediction result, and with the accumulation of forecast experience we can improve the OPM for long-term nature and continuity of prediction.

Beyond the above theories, we design and get the mechanism of OPM as Fig. 1:

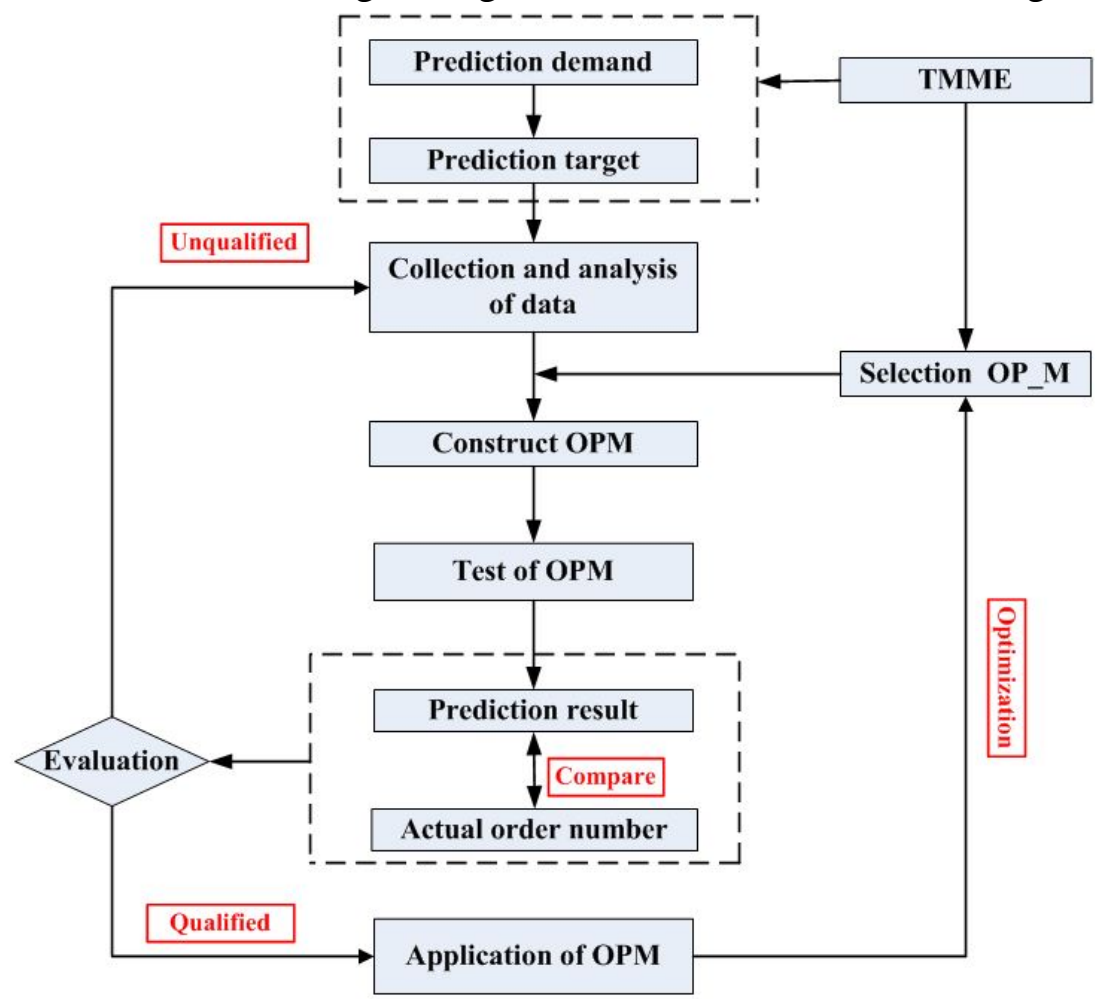

Fig.1 The mechanism of OPM

\section{The data-mining of CD information}

The descriptions of products feature in orders are accurate and specific, but on the contrast is that the customer demand information is ambiguity, diversity, dynamic and contradicted. For the TMME, in order to ensure the accuracy of OPM, the influence caused by customer demand must be considered into the process of prediction. In this paper, at first tracking effective real-time sales data and analyzing the fluctuate trend between customer demand and time, then decomposing the 
customer demand in influencing factors, at last taking distortion adjustment of customer demand by combing the market investigation and feedback information, so it provides the basis for time series decomposition prediction of OPM.According to actual features of TMME, proposing the processing flow of CD which is shown in Fig.2.

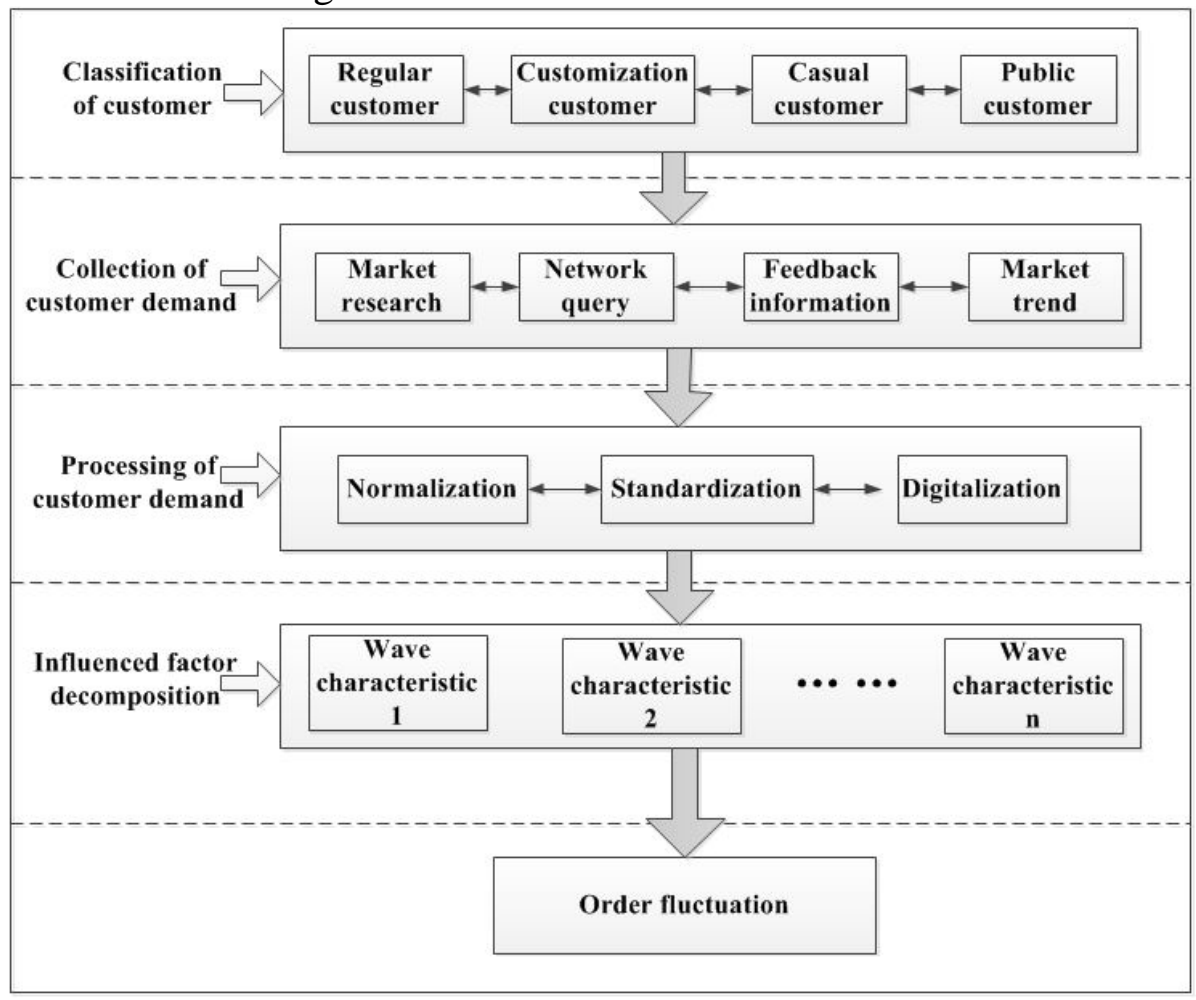

Fig.2 The processing flow of CD

\section{Construct OPM based on improved time series}

According to the different characteristics of CD information we can decompose the influence factors into four aspects: long-term or trend factor $(t f)$, seasonal factor ( $s f$ ), cyclic variation factor $(c f)$, random variation factor $(r f)$. Considering the order is the ultimate embodiment and carrier of $\mathrm{CD}$, we can take $\mathrm{CD}$ as center and divide the general trend of order into different results which is caused by above four kinds factor that is focusing on the data of time series order, finally construct mathematical model (as Eq. 1) and calculated the prediction result (R) by analyzing and processing of the input historical data.

$$
R_{t}=F_{t}(t f, s f, c f, r f)=t f_{t} * s f_{t} * c f_{t} * r f_{t}(1)
$$

The $t f$ doesn't ignored the effect of $s f$ when establishing the regression equation, and the market seasonal change is obvious which affects the accuracy of calculating $t f$, so we must remove the $t f$ during the establishing process. While the $r f$ refers many other different factors and it is often low regularity, in order to avoid resulting more error that we can ignore the calculating of it. And the regulation of $s f$ is usually stable, it can meet the calculating precision by using moving average method. At last we can take the tf,cf and $r f$ as a whole factor that is processed by unification rather than calculating them respectively, and remove the seasonal of order target dependent variables, then construct regression equation as Eq. 2:

$$
R_{t}=s f_{t} *\left(t f_{t} * \mathrm{cf}_{t} * \mathrm{rf}_{t}\right)=s f_{t} *\left(m f_{t}\right)
$$

Where $m f$ represents the mix factor of $t f, c f$ and $r f$, and the calculating flow is shown in Fig.3: 


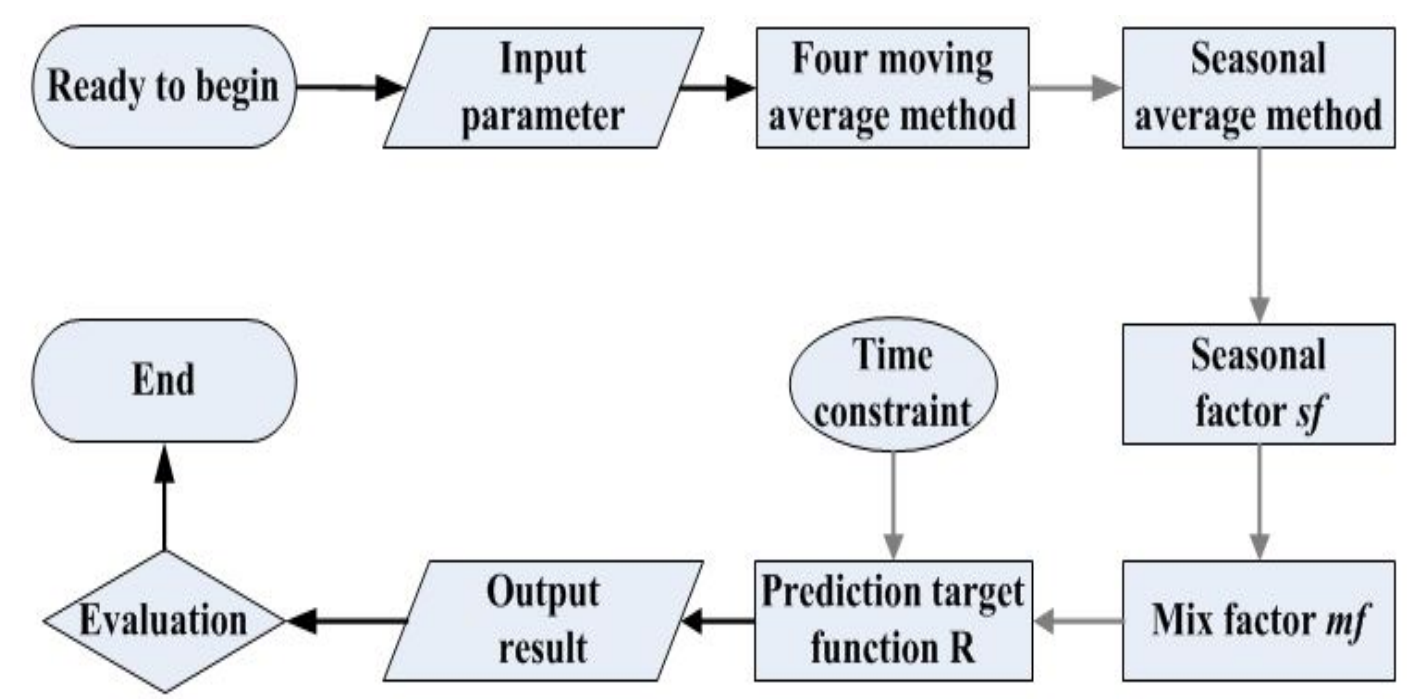

Fig.3 The calculating flow of improved time series

\section{Case analysis}

According to the actual situation of YY-TMME, carries on the example analysis as follows:

Step 1:Collecting to the historical order data for YY-TMME, we organize them into Table 1, and processing the data from 2007 to 2012.And the * represents repetition, the blackbody represents the only result.

Step 2: Using Eviews 6.0to analysis the auto-correlation and partial-correlation of $m f$ as showed in the left of Fig.4, according to the result the model can defined as ARMA $(6,8)$ model, and the graph is showed in the right of Fig.4.

Step3: From the graph we can find the prediction model is meeting our requirement and calculate the $m f$ of year 2013, the values of $m f$ are $(76.08656,77.64300,70.90187,74.58942)$ that are very close to the actual values $(75,81,74,76)$.

Table 1 Processing the historical order data for YY-TMME which from 2007 to 2012

\begin{tabular}{|c|c|c|c|c|c|c|c|c|}
\hline Year & Season & $\begin{array}{c}\text { Order } \\
\text { number }\end{array}$ & $\begin{array}{c}\text { Four } \\
\text { moving } \\
\text { average } \\
\text { method }\end{array}$ & $\begin{array}{c}\text { Center } \\
\text { average }\end{array}$ & $s f^{*} r f$ & $\begin{array}{c}\text { Average } \\
\text { sf }\end{array}$ & $\begin{array}{c}\text { Correct } \\
s f\end{array}$ & $m f$ \\
\hline \multirow{4}{*}{2007} & 1 & 67 & & & & $*$ & * & 66.9531 \\
\hline & 2 & 57 & & & & $*$ & $*$ & 57.1254 \\
\hline & 3 & 66 & 65.25 & 64.8750 & 1.0059 & 0.9967 & 1.0003 & 66.0198 \\
\hline & 4 & 71 & 64.50 & 63.6250 & 1.0138 & 0.9945 & 0.9981 & 70.8651 \\
\hline \multirow{4}{*}{2008} & 1 & 63 & 62.75 & 62.0000 & 1.0120 & 0.9957 & 0.9993 & 62.9559 \\
\hline & 2 & 51 & 61.25 & 59.1250 & 1.0359 & 0.9985 & 1.0022 & 51.1122 \\
\hline & 3 & 60 & 57.00 & 57.6250 & 0.9892 & $*$ & $*$ & 60.0180 \\
\hline & 4 & 54 & 58.25 & 59.8750 & 0.9729 & $*$ & $*$ & 53.8974 \\
\hline \multirow{4}{*}{2009} & 1 & 68 & 61.50 & 62.8750 & 0.9781 & $*$ & $*$ & 67.9524 \\
\hline & 2 & 64 & 64.25 & 65.5000 & 0.9808 & $*$ & $*$ & 64.1408 \\
\hline & 3 & 71 & 66.75 & 68.0000 & 0.9816 & $*$ & $*$ & 71.0213 \\
\hline & 4 & 64 & 69.25 & 70.2500 & 0.9858 & $*$ & * & 63.8784 \\
\hline \multirow{4}{*}{2010} & 1 & 78 & 71.25 & 71.6250 & 0.9948 & $*$ & $*$ & 77.9454 \\
\hline & 2 & 72 & 72.00 & 73.0000 & 0.9863 & $*$ & $*$ & 72.1584 \\
\hline & 3 & 74 & 74.00 & 72.6250 & 1.0189 & $*$ & $*$ & 74.0222 \\
\hline & 4 & 72 & 71.25 & 70.8750 & 1.0053 & $*$ & $*$ & 71.8632 \\
\hline \multirow{3}{*}{2011} & 1 & 67 & 70.50 & 70.2500 & 1.0036 & $*$ & $*$ & 66.9531 \\
\hline & 2 & 69 & 70.00 & 70.2500 & 0.9964 & $*$ & $*$ & 69.1518 \\
\hline & 3 & 72 & 70.50 & 71.3750 & 0.9877 & $*$ & $*$ & 72.0126 \\
\hline
\end{tabular}




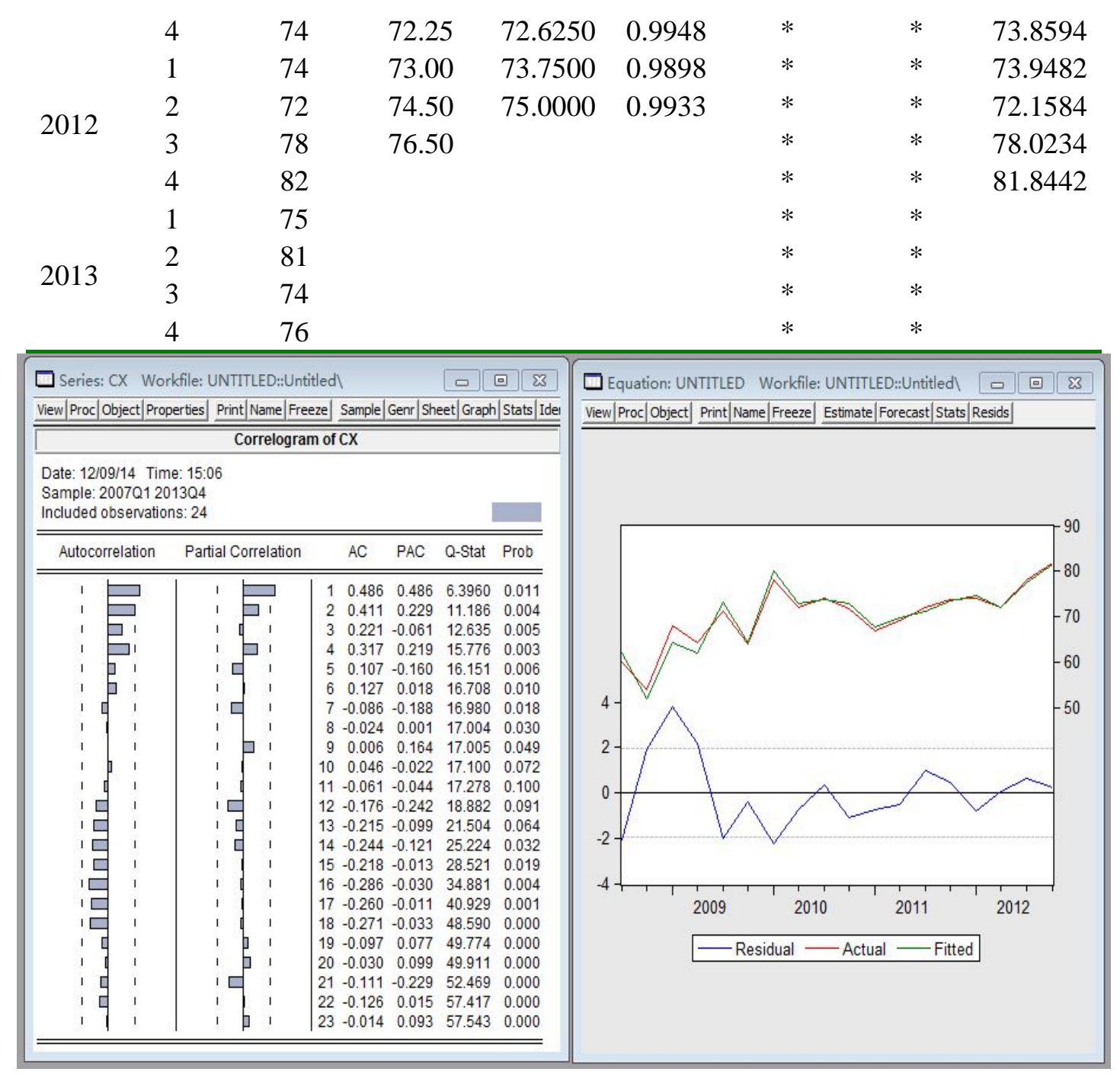

Fig.4 The analysis and comparison of $m f$

\section{Conclusion}

Through the application of OPM we can get a satisfactory result, but because of the number of samples is less which resulting in the accuracy of prediction model is not high. In order to improve the prediction accuracy, not only we need to consider the CD, but also some other related factors, such as the products' upgrading, enterprise transformation etc. At the same time we should adopt some optimization method to optimize the OPM.

\section{Acknowledgements}

This work was financially supported by the National Nature Science Fund Project of China (71171154), and the Fundamental Research Funds for the Central Universities (2014-zy-062).

\section{References}

[1] Lundholm, R.J., S.E. McVay and A. Curtis, Forecasting Sales: A Model and Some Evidence from the Retail Industry [J]. SSRN Working Paper Series, 2012.

[2] Wang C. Research of Order Forecast and Inventory Optimization of Aeronautical Manufacturing Industry [D]. Shanghai Jiao Tong University, 2012.

[3] Liu C.X. Combination Prediction Research of Electronic Products Sales based on CPFR [D]. Hunan University, 2011.

[4] Gong R.R. Order Prediction Research of RBF Enterprise based on OLS and EPSO [J].Computer 
Engineering and Application, 243(2011)224-226.

[5] Wang J.L, Kuo J.H, Chou S.Y, etc. A comparison of bullwhip effect in a single-stage supply chain for auto correlated demands when using Correct, MA, and EWMA methods [J]. Expert Systems with Applications, 37(2010)4726-4736.

[6] Gao J.J, Tan C.L. A Support Vector Machine Method of Product DemandForecasting [J].Journal of Shanghai University (Natural Science Edition), 15(2009) 71-76.

[7] Dong X.H, Li C.L. Research of GP Algorithm and Implementation of System of Machine Products Sales Forecasting [J].Manufacturing Automation, 32(2010)10-12. 